\title{
Optimization of the confinement energy of quantum-wire states in T-shaped GaAs/AlxGa1-XAs structures
}

\author{
Langbein, Wolfgang Werner; Gislason, Hannes; Hvam, Jørn Märcher
}

Published in:

Physical Review B

Link to article, DOI:

10.1103/PhysRevB.54.14595

Publication date:

1996

Document Version

Publisher's PDF, also known as Version of record

Link back to DTU Orbit

Citation (APA):

Langbein, W. W., Gislason, H., \& Hvam, J. M. (1996). Optimization of the confinement energy of quantum-wire states in T-shaped GaAs/AlxGa1-xAs structures. Physical Review B, 54(20), 14595-14603.

https://doi.org/10.1103/PhysRevB.54.14595

\section{General rights}

Copyright and moral rights for the publications made accessible in the public portal are retained by the authors and/or other copyright owners and it is a condition of accessing publications that users recognise and abide by the legal requirements associated with these rights.

- Users may download and print one copy of any publication from the public portal for the purpose of private study or research.

- You may not further distribute the material or use it for any profit-making activity or commercial gain

- You may freely distribute the URL identifying the publication in the public portal 


\title{
Optimization of the confinement energy of quantum-wire states in T-shaped GaAs/Al ${ }_{x} \mathbf{G a}_{1-x}$ As structures
}

\author{
W. Langbein, H. Gislason, and J. M. Hvam \\ Mikroelektronik Centret, The Technical University of Denmark, Building 345 East, DK-2800 Lyngby, Denmark
}

(Received 7 February 1996; revised manuscript received 16 July 1996)

\begin{abstract}
We report on an optimization of the wire confinement energies of the confined electronic states at the T-shaped intersection of GaAs and $\mathrm{Al}_{x} \mathrm{Ga}_{1-x} \mathrm{As}$ quantum wells. These structures can be produced by the cleaved edge overgrowth technique. We present an analytical model for the confinement to give insight into the basic mechanism. The optimization of the confinement energy is done by calculations in a six-band $\mathbf{k} \cdot \mathbf{p}$ approximation for the valence band and in an isotropic effective-mass approximation for the conduction band. The confined valence-band states are only weakly bound at the T-shaped intersection due to the large and anisotropic hole effective masses. Employing optimized sample parameters, confinement energies for the free-electron-hole pairs are nearly doubled compared to symmetric structures, and $34 \mathrm{meV}$ are predicted for a 3-nm overgrown GaAs well. This is expected to be further enhanced by the Coulomb interaction, that is neglected in the numerical model. The experimental structures grown using the optimized geometry show wire confinement energies of up to $54 \mathrm{meV}$, which is significantly larger than $K T$ at room temperature and larger than previously reported. [S0163-1829(96)04843-6]
\end{abstract}

\section{INTRODUCTION}

The fabrication of semiconductor nanostructures with an effective dimensionality lower than 2 is an active field of research in semiconductor physics. The decreasing dimensionality leads to a concentration of the density of states at the band edge and to a reduced scattering in the remaining directions of free motion, which is of interest for optical and electronic devices such as laser diodes and fast transistors. The reduction in the effective dimensionality can be achieved in structures of sizes in the nanometer range, in which the energy difference of the confined states is larger than both the thermal energy and the excitonic binding energy.

Technologically, the step from the two-dimensional quantum-well (QW) structures, which are fabricated by thinfilm growth, down to one dimension is complicated. The lateral structuring of the thin films by lithographic methods is limited to an accuracy of some ten nanometers, and thus gives rise to a strong inhomogeneous broadening of the electronic properties. This may be circumvented by selforganized growth in prepatterned materials, such as etched $V$ grooves on GaAs substrates. ${ }^{1}$

Another possibility is the fabrication of a T-shaped intersection of two QW's, as was proposed by Chang, Chang, and Esaki. ${ }^{2}$ These structures can be realized by the cleaved edge overgrowth technique. ${ }^{3-7}$ Using the latter technique, both dimensions are controlled by the high accuracy of the molecular-beam epitaxy (MBE). At the intersection, a bound quantum-wire state (TW state) forms. The photoluminescence of these structures, attributed to the TW-state transition, was demonstrated to originate from the $\mathrm{T}$ intersections by optical near-field spectroscopy. ${ }^{8,9}$ Optically and electrically pumped lasing of the TW transition in these structures was observed and attributed to excitonic processes. ${ }^{10-12}$ The largest wire confinement energy (WCE) of a TW state re- ported up to now is $38 \mathrm{meV} .{ }^{13}$ A theoretical calculation of the WCE in the T-shaped structures for various sample designs has not yet been published. Hence an optimization of the design has not been achieved.

In this paper, we present a calculation of the electronic states confined at the T-shaped intersection of two QW's, the TW states. For given overgrown QW widths, we optimize the sample design for the maximal WCE by the variation of width and Al content of the (001) multiple QW (MQW). We show that this can improve the WCE compared to symmetric T-shaped structures by a factor of 2, giving a calculated WCE of $34 \mathrm{meV}$ for an overgrown 3-nm (110) QW, without consideration of the Coulomb interaction. These predictions are confirmed by the properties of an experimental structure, which shows a WCE of $54 \mathrm{meV}$.

\section{THEORETICAL MODELS}

To optimize the sample parameters for maximum WCE prior to the growth, we employ a calculation of the TW states. First we present an analytical model to give the basic confinement mechanism that increases the WCE in asymmetric structures compared to symmetric ones. Then we describe the quantitative numerical model used for the optimization of the T-shaped structures and present the results.

\section{A. Analytical model}

In order to gain insight into the confinement mechanism of the electronic states at the T-shaped intersection, we first discuss an analytical model for the WCE, which is schematically depicted in Fig. 1. An infinite barrier height and particles with an isotropic effective mass $m$ are assumed. The quantization energy $E_{\mathrm{ib}}$ of the lowest state in the overgrown QW of the width $L_{[110]}$ at the barrier regions of the cleaved QW [Fig. 1(a)] accordingly reads 
a)

b)

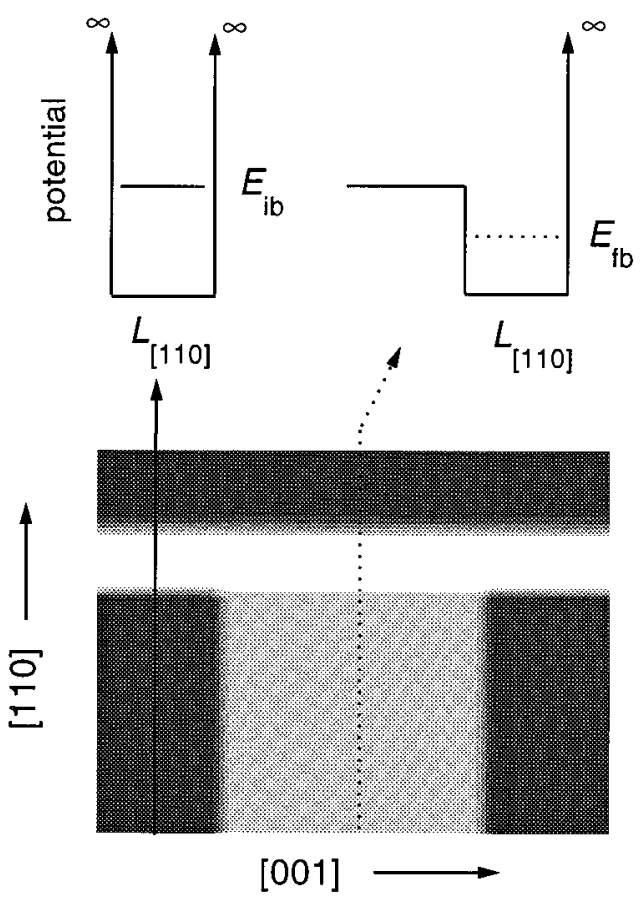

c)

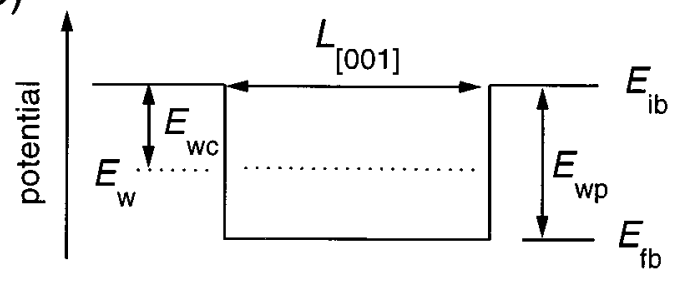

FIG. 1. Schematic drawing of an asymmetric T-shaped structure with the relevant potentials and quantization energies used in the analytical model.

$$
E_{\mathrm{ib}}=\frac{\hbar^{2} \pi^{2}}{2 m L_{[110]}^{2}} .
$$

The largest energy separation between the TW transition and the lowest transitions in the (001) and (110) QW's is obtained when the transition energies of both types of QW's are equal. Any difference between the two QW transition energies reduces the relative WCE to the lowest of them, because the TW state is always a mixture of both QW states. In such a structure with equal $\mathrm{QW}$ transition energies, one barrier of the overgrown (110) QW is lowered down to $E_{\mathrm{ib}}$ in the well region of the cleaved (001) QW [Fig. 1(b)]. To calculate the quantization energy $E_{\mathrm{fb}}$ in this asymmetric quantum well, we use the one-dimensional Schrödinger equation

$$
\left[\frac{\hbar}{2 m} \nabla_{z}^{2}+V(z)-E\right] \Psi(z)=0
$$

This gives a reduced quantization energy $E_{\mathrm{fb}}$ of $0.542 E_{\mathrm{ib}}$ due to the penetration of the state into the barrier of finite height. The two different quantization energies $E_{\mathrm{fb}}$ and
$E_{\mathrm{ib}}$ for the states in the overgrown QW in the well and barrier regions of the (001) QW form a one-dimensional confinement potential of the magnitude $E_{\mathrm{wp}}=E_{\mathrm{ib}}-E_{\mathrm{fb}}$ $\approx 0.458 E_{\mathrm{ib}}$ in the overgrown $\mathrm{QW}$ along the [001] direction [Fig. 1(c)].

Using Eq. (2), we determine the quantization energies of the TW states in the [001] direction using the confinement potential $E_{\mathrm{wp}}$. For a symmetric T-shaped wire structure, e.g., where the width $L_{[001]}$ of the $(001) \mathrm{QW}$ is equal to the width $L_{[110]}$ of the overgrown $(110) \mathrm{QW}$, this results in a TW state with a quantization energy of $0.481 E_{\mathrm{wp}}$ and thus to an absolute energy of $E_{w} \approx E_{\mathrm{fb}}+0.481 E_{\mathrm{wp}} \approx 0.762 E_{\mathrm{ib}}$. The WCE $E_{\mathrm{wc}}$ to the wire barrier $E_{\mathrm{ib}}$ is then given by $0.238 E_{\mathrm{ib}}$. If we increase $L_{[001]}$ until the second TW state is bound in the [001] direction, which occurs for $L_{[001]} \approx 1.48 L_{[110]}$, the reduction of the wire quantization energy along the [001] direction leads to an enhancement of the WCE to $E_{\mathrm{wc}} \approx 0.296 E_{\mathrm{ib}}$ at this optimum (001) QW width.

This simple model thus predicts a binding energy of $24 \%$ of the QW quantization energy for the symmetric structure, and of $30 \%$ for the optimized structure. The results of the following numerical calculation show a confinement energy of $12-16 \%$ for the symmetric structure and about $22-30 \%$ for the optimized structure. The better agreement for the optimized structure is due to the larger ratio between $L_{[001]}$ and $L_{[110]}$, for which the simplified analytical model is better suited. Also, the relative WCE drops in the realistic structures with decreasing well width due to the finite barrier height.

\section{B. Numerical model}

To obtain a quantitative prediction of the dependence of the WCE in T-shaped structures on the various sample parameters, we have implemented a numerical model for the electronic states in such structures. It treats the $\Gamma$ conduction-band states in an isotropic effective-mass approximation and the valence-band states in a six-band $\mathbf{k} \cdot \mathbf{p}$ model, thus including the mixing of heavy hole, light hole, and split-off hole. Such a treatment has been used successfully to describe the properties of quantum wells ${ }^{14,15}$ and to calculate the states in model rectangular quantum wires. ${ }^{16}$ Coulomb interactions are neglected in the present calculations. However, the excitonic binding energy in the TW states $^{17,18}$ is reported to be larger than in the QW states, ${ }^{2,19,20}$ thus leading to an additional confinement of the TW exciton states.

The TW states are calculated for an array of T-shaped intersections, allowing for periodic boundary conditions. The array period along the [001] direction is given by the period of the cleaved MQW structure. In the overgrowth direction [110], one end of the unit cell is given by the surface of the overgrown barrier, while the other end has to be introduced artificially in a specific depth of the (001) MQW. The depth is chosen to be large enough so that it does not have a significant influence on the TW states. The unit cells used in the calculations are displayed together with the wave functions of the TW states in Figs. 3, 4, 7, and 6, that will be discussed below. 
To calculate the electronic subbands, we start with a determination of the subband wave functions $\Phi^{n}$ at the zone center $(\Gamma)$ in an effective mass approach. We discretize one period of the structure in real space to $N_{\mathbf{x}} \times N_{\mathbf{y}}$ points in the directions $\mathbf{x} \equiv[001]$ and $\mathbf{y} \equiv[110]$, where the numbers $N_{\mathbf{x}, \mathbf{y}}$ are powers of 2 . For the presented results, we used $N_{\mathbf{x}, \mathbf{y}}=32$.

In the following, we describe the calculation for onedimensional superlattices in the $\mathrm{z}$ direction, for simplicity. The expansion to the two-dimensional case is straightforward.

As an ansatz for the $\Phi^{n}$, we use the Fourier components $(\cos , \sin )\left[\left(2 \pi n_{\mathbf{z}} / L_{\mathbf{z}}\right) z\right]$ with $n_{\mathbf{z}}=0,1, \ldots, N_{\mathbf{z}} / 2$, excluding the sine components of the lowest and highest momenta, which vanish on the grid in real space. Using the currentconserving boundary conditions, this leads to the eigenvalue equation

$$
\begin{aligned}
& \left(\nabla_{z} \frac{\hbar^{2}}{2 m_{\mathrm{z}}(z)} \nabla_{z}+V(z)-E_{n}\right) \\
& \times \underbrace{\left[\sum_{n_{\mathrm{z}}=0}^{N_{z} / 2} c_{n_{\mathrm{z}}}^{n} \cos \left(\frac{2 \pi n_{\mathrm{z}}}{L_{\mathrm{z}}} z\right)+s_{n_{\mathrm{z}} \neq\left\{0, N_{\mathrm{z}} / 2\right\}}^{n} \sin \left(\frac{2 \pi n_{\mathrm{z}}}{L_{\mathbf{z}}} z\right)\right]}_{\Phi^{n}(z)}=0,
\end{aligned}
$$

with the superlattice period $L_{\mathbf{z}}$, the superlattice potential $V_{\mathbf{z}}$, the effective mass $m_{\mathbf{z}}(z)$, and the eigenenergies $E_{n}$ for the eigenvectors $\left(c^{n}, s^{n}\right)$.

To determine the conduction subband dispersion around the $\Gamma$ point, we use the $M_{\mathbf{z}}(10-100)$ lowest eigenstates $\Phi^{n}(z)$ of Eq. (3) as an ansatz for the eigenstates $\Psi^{n}(\vec{k}, z)$ with the quasimomentum $\hbar \vec{k}$ :

$$
\begin{aligned}
& \left(\vec{\nabla} \frac{\hbar^{2}}{2 m_{\mathrm{e}}(z)} \vec{\nabla}+V^{\mathrm{e}}(z)-E_{n}^{\mathrm{e}}(\vec{k})\right) \\
& \times \underbrace{\left[e^{i \vec{k} \vec{x}}\left(\sum_{m=1}^{M_{\mathrm{z}}} a_{m}^{n, \vec{k}} \Phi^{m}(z)\right)\right]}_{\Psi^{n}(\vec{k}, z)}=0 .
\end{aligned}
$$

Here $m_{\mathbf{e}}$ is the isotropic conduction-band mass, and the potential $V^{\mathbf{e}}(z)$ is given by the band offsets in $\mathrm{Al}_{x} \mathrm{Ga}_{1-x}$ As. The eigenvectors $\mathbf{a}^{n, \vec{k}}$ define the eigenstates $\Psi^{n}(\vec{k}, z)$ with the energy $E_{n}^{\mathbf{e}}(\vec{k})$, which are the envelope functions of the $n$th conduction subband with the quasimomentum $\hbar \vec{k}$.

To determine the valence subband states, we start with Eq. (3) using the mean effective mass $1 / m_{\mathbf{z}}(z)=\gamma_{1}(z)$ of the valence bands and the potential $V(z)$ of the heavy-hole band. We use the resulting eigenstates $\Phi^{n}(z)$ as an ansatz for the periodic part of the envelope functions of the six spin-orbitcoupled components $|\mu\rangle$ of the valence band, ${ }^{21,22}$

$$
\begin{aligned}
\sum_{\mu^{\prime}=1}^{6} & \left\{T_{\mu, \mu^{\prime}}(z)+\left[V_{\mu}^{\mathbf{h}}(z)-E_{n}^{\mathbf{h}}\right] \delta_{\mu, \mu^{\prime}}\right\} \\
\times & \underbrace{\left[e^{i \vec{k}}\left(\sum_{m=1}^{M_{\mathbf{z}}} g_{m}^{n, \mu^{\prime}, \vec{k}} \Phi^{m}(z)\right)\right]}_{\Psi_{\mu^{\prime}}^{n}(\vec{k}, z)}=0, \quad \forall_{\mu^{\prime}}
\end{aligned}
$$

The eigenvectors $\mathbf{g}^{n, \vec{k}}$ then describe the valence subband envelope function on the $\Phi(z)$ basis. The kinetic operators $T_{\mu, \mu^{\prime}}(z)$ and the potential $V_{\mu}^{\mathrm{h}}(z)$ are given by the Luttinger Hamiltonian $^{22}$ and the valence-band offsets in $\mathrm{Al}_{x} \mathrm{Ga}_{1-x}$ As. In $T_{\mu, \mu^{\prime}}$, we replace the quasimomentum $\hbar \vec{k}$ by the corresponding operator $-i \hbar \vec{\nabla}$. To obtain a Hermitian Hamiltonian even with spatially varying Luttinger parameters $\gamma_{1,2,3}(z)$, we symmetrize the operators $T_{\mu, \mu^{\prime}}(z) .^{23}$.

Solving Eq. (5) on the product basis of the envelope functions and the spin-orbit coupled zone-center states of the valence band $|\mu\rangle$, gives the subband wave functions $\Sigma_{\mu} \Psi_{\mu}^{n}(\vec{k}, z)|\mu\rangle$ for every subband state $n$ and quasimomentum $\hbar \vec{k}$.

We now consider the optical transition matrix element $P_{\epsilon}$ between the $l$ th $\Gamma$ conduction subband $\Psi^{l}(\vec{k}, z)|0,0\rangle\left|s_{\mathrm{e}}\right\rangle$ and the $m$ th valence subband $\Sigma_{\mu} \Psi_{\mu}^{m}(\vec{k}, z)|\mu\rangle$ for the light polarization $\vec{\epsilon}$. It can be written down using the representation of the spin-orbit-coupled basis $|\mu\rangle$ in the uncoupled basis $(|X\rangle,|Y\rangle,|Z\rangle)\left|s_{h}\right\rangle$, with the electron and hole spins $s_{\mathbf{e}}$ and $s_{\mathbf{h}}$, and the zone-center valence-band-orbital states $|X\rangle,|Y\rangle,|Z\rangle$.

$$
\begin{aligned}
P_{\vec{\epsilon}}^{l, m}(\vec{k}) \propto \sum_{\mu=1}^{6} \vec{\epsilon} \cdot\left\langle s_{\mathrm{e}}\right|\left\langle 0,0\left|\Psi^{l}(\vec{k}, z)\right| \vec{\nabla}\left|\Psi_{\mu}^{m}(\vec{k}, z)\right| \mu\right\rangle \\
=\sum_{\mu=1}^{6} \underbrace{\left[\frac{L_{\mathbf{z}}}{N_{\mathbf{z}}} \sum_{n_{\mathbf{z}}=0}^{N_{\mathbf{z}}-1} \Psi^{l}\left(\vec{k}, \frac{n_{\mathbf{z}}}{N_{\mathbf{z}} L_{\mathbf{z}}}\right) \cdot \Psi_{\mu}^{m}\left(\vec{k}, \frac{n_{\mathbf{z}}}{N_{\mathbf{z}} L_{\mathbf{z}}}\right)\right]}_{\text {envelope overlap }}
\end{aligned}
$$

$$
\times\left(\sum_{s_{\mathbf{h}}=-1 / 2}^{1 / 2} \delta\left(s_{\mathrm{e}}, s_{\mathbf{h}}\right) \sum_{P \in\{X, Y, Z\}} C_{s_{\mathbf{h}}, P}^{\mu} \vec{\epsilon} \cdot\langle S|\vec{\nabla}| P\rangle\right) .
$$

$$
\text { dipole matrix element }
$$

Here, $C_{s_{\mathbf{h}}, P}^{\mu}$ are the coefficients relating the spin-orbit coupled basis $|\mu\rangle$ to the uncoupled basis $|P\rangle\left|s_{\mathbf{h}}\right\rangle$. The momentum matrix elements $\left\langle S\left|\nabla_{x}\right| X\right\rangle,\left\langle S\left|\nabla_{y}\right| Y\right\rangle$, and $\left\langle S\left|\nabla_{z}\right| Z\right\rangle$ are equal and given in Ref. 24; all other combinations vanish.

In order to calculate the interband absorption, we determine the subband states and energies on an equidistant grid of the quasimomentum $\hbar \vec{k}$ within the first mini-Brillouin zone of the superlattice. To determine the density of states (DOS) as a function of energy $D(E)$, we discretize the en- 
TABLE I. material parameters of $\mathrm{Al}_{x} \mathrm{Ga}_{1-x}$ As used in the calculations are from Properties of Aluminum Gallium Arsenide, edited by S.Adachi (INSPEC, London, 1988).

\begin{tabular}{lccc}
\hline \hline Symbol & Parameter & Unit & $\mathrm{Ga}_{1-x} \mathrm{Al}_{x} \mathrm{As}$ \\
\hline$E_{g}$ & energy gap & $(\mathrm{eV})$ & $1.519+1.138 x+0.47 x^{3}$ \\
$\Delta E_{c} / \Delta E_{v}$ & band offsets & & $67 / 33$ \\
$m_{e}$ & electron mass & $\left(\mathrm{m}_{0}\right)$ & $1 /(14.9-8.2 x)$ \\
$\gamma_{1}$ & Luttinger & & $7.1-3.34 x$ \\
$\gamma_{2}$ & parameter & & $2.02-1.12 x$ \\
$\gamma_{3}$ & & & $2.91-1.49 x$ \\
$\Delta_{\text {so }}$ & spin-orbit splitting & $(\mathrm{eV})$ & $0.34-0.065 x$ \\
\hline \hline
\end{tabular}

ergy into equidistant $\Delta E$ segments. The same is done with the quasimomentum space, in which the DOS is constant. By adding the DOS of every cube in quasimomentum space to the corresponding energy segment, we obtain $D(E)$.

To obtain a sufficient statistics, we have to divide the mini-Brillouin zone into millions of cubes. The corresponding eigenenergies are obtained by a three-dimensional spline interpolation in between the calculated eigenenergies at the grid points in quasimomentum space, including the correct boundary conditions at the zone center and the zone edge. To obtain the joint DOS for the interband absorption, we use the same procedure, but we also interpolate the optical transition matrix elements for the three polarization directions of light [Eq. (6)].

\section{NUMERICAL RESULTS AND DISCUSSION}

We now present results of the numerical calculations, which give a quantitative description of the WCE's and polarization of the TW transition. The $\mathrm{Al}_{x} \mathrm{Ga}_{1-x} \mathrm{As}$ material parameters used in the calculations are listed in Table I.

\section{A. Symmetric structures}

In Fig. 2 we display the calculated transition energies (a), the WCE (b), and the optical anisotropy (c) of the TW transition in symmetric GaAs T-shaped structures with $\mathrm{Al}_{0.3} \mathrm{Ga}_{0.7} \mathrm{As}$ barriers, as a function of well width. The corresponding values for a (110) $\mathrm{QW}$ are plotted for comparison.

The TW transition energy increases with decreasing well width due to the increasing quantization energies. In both the conduction and valence bands, the WCE amounts to $12-16 \%$ of the (110) QW quantization energy. The lower relative confinement in the thinner structures is due to the finite barrier height. This leads for 3-nm well width to a WCE of 17 $\mathrm{meV}$ in the conduction band and $4 \mathrm{meV}$ in the valence band. The TW transition energy is accordingly redshifted by 21 meV compared to the (110) QW transition. The calculated WCE's in the conduction band are in agreement with the results of Wegscheider et al. ${ }^{7}$ It should be noted here that, due to the anisotropic hole mass, the hole quantization energy and thus the transition energy is lower in (110) QW's compared to (001) QW's of equal thickness. ${ }^{25,26}$ Additionally, the highest valence-band states in the (001) and (110)

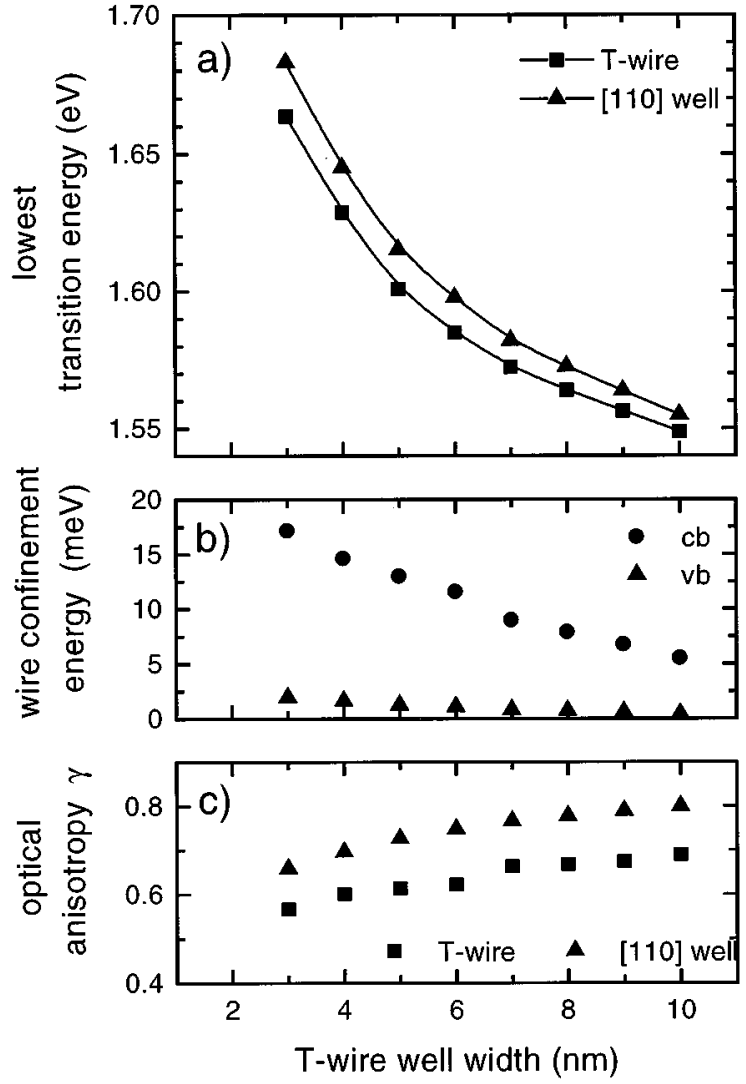

FIG. 2. Results of the numerical model for symmetric T-shaped wire structures with $\mathrm{GaAs}$ wells and $\mathrm{Al}_{0.3} \mathrm{Ga}_{0.7} \mathrm{As}$ barriers as a function of the well width. (a) Transition energy of the lowest wire state (cb1-vb1) and of the lowest transition in a (110) QW with equal barriers. (b) Wire confinement energies for the conduction- and valence-band states. (c) The optical anisotropy $\gamma=P_{[001]} / P_{[1 \overline{1} 0]}$ of the TW transition and the lowest (110) QW transition.

QW's consist of different mixtures of the heavy- and lighthole states, which leads to an inefficient localization at the intersection.

The optical anisotropy $\gamma$ of the TW transition [Fig. 2(c)] is given by the ratio between the transition strengths for the light polarizations orthogonal and parallel to the wires, e.g., $\gamma=P_{[001]} / P_{[1 \overline{1} 0]}$, with the dipole transition strengths $P_{[h k l]}$ for the polarization directions $[h k l]$. It is lower than that of the lowest transition in the (110) QW. The TW transition is slightly allowed for the polarization along the overgrowth direction [110] (not shown), in contrast to the forbidden character of the lowest transition in the (110) QW for this polarization. This redistribution of transition strength from $P_{[001]}$ to $P_{[110]}$ shows the participation of the $(001)$ QW states in the TW state. The close similarity of the anisotropy of the TW transition and of the (110) QW transition is due to the dominance of the lowest (110) QW valence subband in the TW valence-band state.

The envelope functions of the conduction- and valenceband TW states in a symmetric T-shaped structure of 3-nm QW width are shown in Fig. 3. The conduction-band TW state is confined symmetrically at the intersection, while the valence-band TW state is more extended along the overgrown (110) QW. This is a direct consequence of the isotro- 


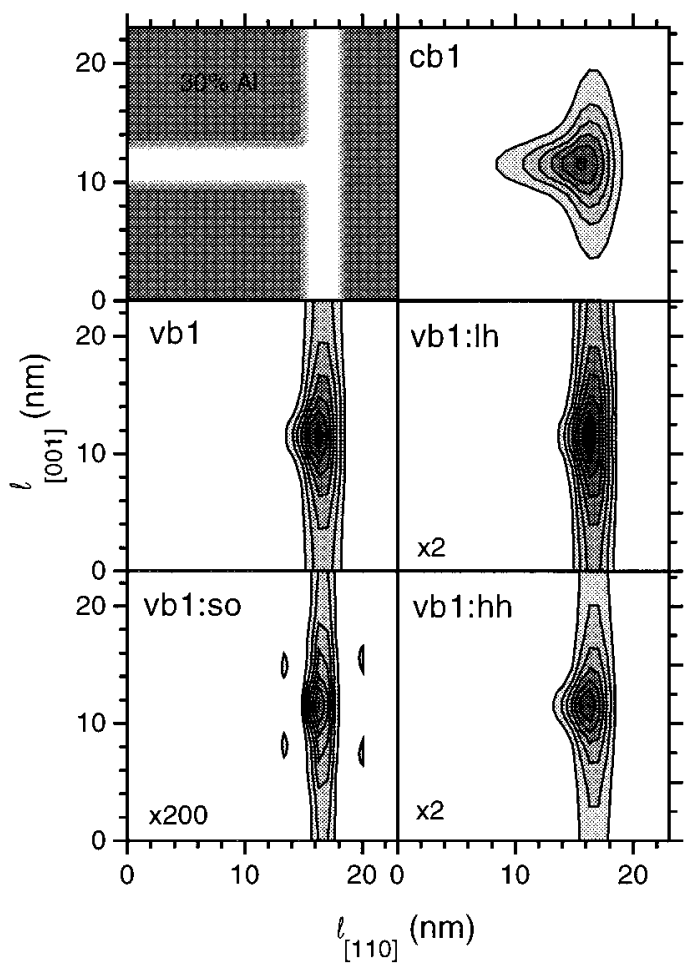

FIG. 3. Confined zone-center electronic states in a T-shaped structure consisting of a 3-nm GaAs/20-nm Al $\mathrm{Al}_{0.3} \mathrm{Ga}_{0.7} \mathrm{As}$ (001) MQW overgrown by a 3-nm (110) GaAs QW and an $\mathrm{Al}_{0.3} \mathrm{Ga}_{0.7} \mathrm{As}$ barrier. The shape of the structure is depicted in the left upper picture. The contours of the constant probability are plotted for the conduction-band TW state (cb1) and the valence-band TW state (vb1) with its constituents of heavy hole (hh), light hole (lh), and split-off hole (so). The scaling factors between the plots are given.

pic conduction-band mass and the anisotropic valence-band mass. The split-off band contributes only weakly to the TW state even for this narrow structure due to the large spin-orbit splitting.

\section{B. Asymmetric structures with GaAs wells}

To optimize the WCE in T-shaped structures with GaAs wells, the (110) QW width has to be taken to be somewhat smaller than the (001) QW width to compensate for the higher hole mass in the [110] direction. This was verified experimentally by Someya and co-workers for structures with 5.4-nm GaAs (001) QW's and several overgrown (110) GaAs QW widths between 4.5 and $8 \mathrm{~nm} .{ }^{18,27}$ A maximum of the WCE was found at a (110) QW width, for which the (001) and (110) QW transition energies are matched.

We have calculated the transitions in the structures grown by Someya and co-workers, ${ }^{18,27}$ and compare our results for the transition energies, optical anisotropy and wave functions of the TW state in Fig. 4 with their experimental results.

We find the calculated transition energies to be in good agreement with the experimental values. This seems to be fortunate, because the exciton binding energy is not taken into account in the calculation. At the crossover of the (001) and (110) QW transition energies [Fig. 4(a)], the (110) QW width of $5.0 \mathrm{~nm}$ is slightly smaller than the (001) QW width of $5.4 \mathrm{~nm}$ due to the valence-band anisotropy. We find the highest WCE at this position, as in the experiment. The cal- a)

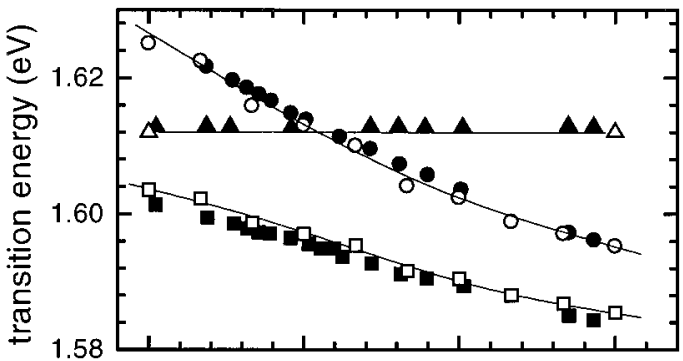

b)

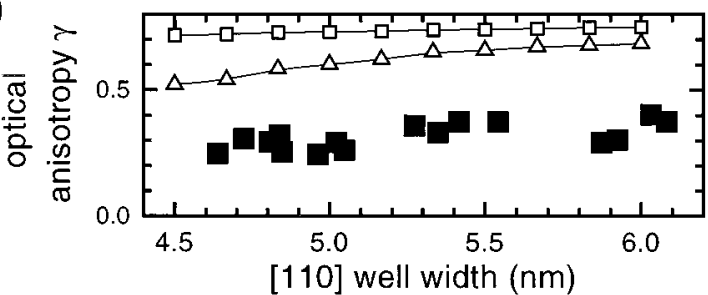

c)

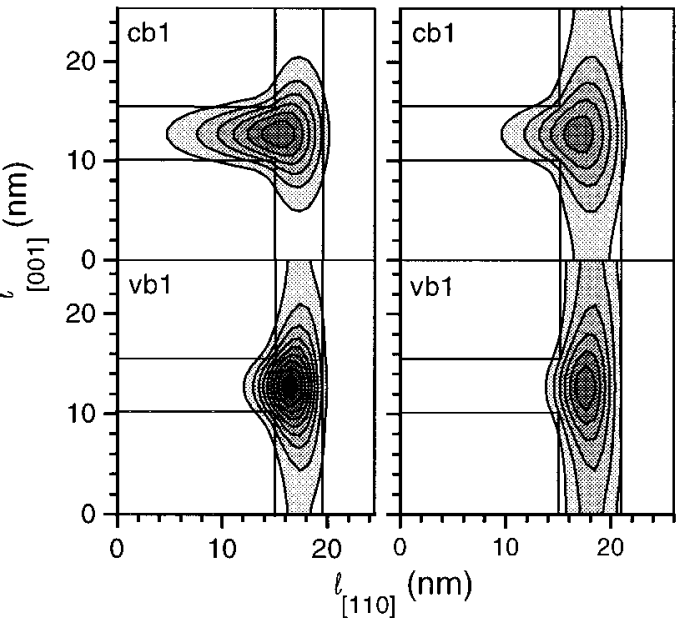

FIG. 4. Experimental and calculated results for asymmetric T-shaped structures consisting of a 5.4-nm GaAs/20-nm $\mathrm{Al}_{0.3} \mathrm{Ga}_{0.7} \mathrm{As}(001) \quad \mathrm{MQW}$ and $\mathrm{GaAs} / \mathrm{Al}_{0.3} \mathrm{Ga}_{0.7} \mathrm{As}(110)$ QW's of widths between 4.5 and $6 \mathrm{~nm}$. The experimental results are taken from Refs. 18 and 27. (a) Transition energies of the (001) QW (triangles), the (110) QW's (circles) and the TW state (squares). Experimental data (full symbols) and calculations (empty symbols and lines) are shown. (b) Optical anisotropy $\gamma=P_{[001]} / P_{[1 \Gamma 0]}$ of the TW transition. The experimental data (squares) are compared to the calculated data for the TW transition (open triangles) and the (110) QW transition (open squares). (c) TW states for a 4.5-nm (110) QW width (left) and 6-nm (110) QW width (right). The contours of the constant probability are plotted for the TW conductionband state $(\mathrm{cb})$ and TW valence-band state $(\mathrm{vb})$. All contours are on an equal scale. The T-shaped structure is indicated by lines.

culated WCE's are slightly smaller than the experimental ones. This might be due to a higher exciton binding energy of the TW state. ${ }^{18}$ The calculated exciton binding energy of the TW state, determined using the calculated dispersion along the $[1 \overline{1} 0]$ direction and the wave functions of the TW states, is between 12 and $10 \mathrm{meV}$. This is comparable to the exciton binding energy in the QW's, and thus no significant additional confinement is predicted. However, the valenceband TW state is only weakly bound, and, thus, the calculated binding energy, using only the one confined valenceband TW state, is not very accurate, because the Coulomb interaction mixes different valence-band states. 

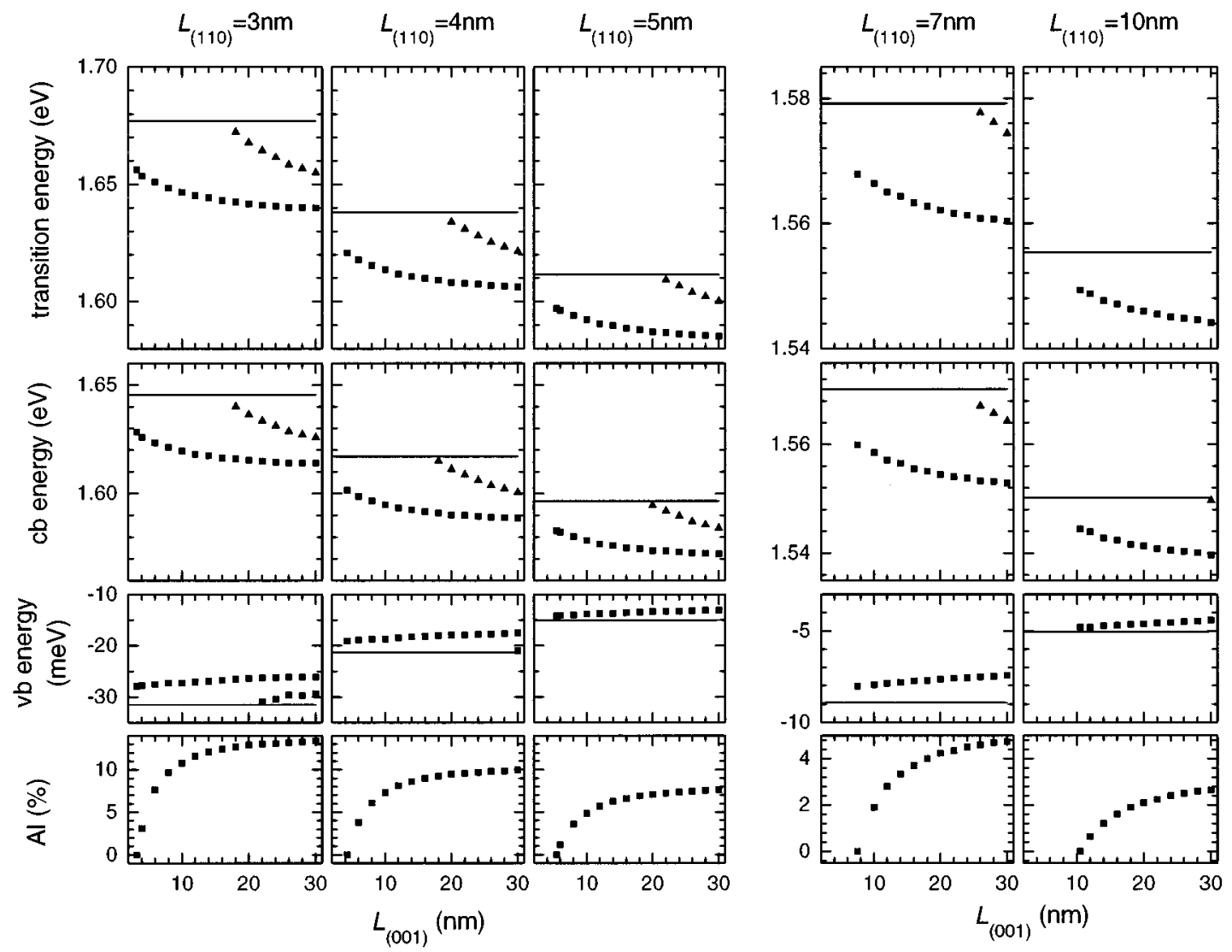

FIG. 5. Transition energies and energies of the conduction- and valence-band TW states in T-shaped structures with overgrown (110) QW's of widths 3, 4, 5, 7, and $10 \mathrm{~nm}$. The width of the (001) QW is varied together with the aluminum content, as given in the lowest graphs. The corresponding energies of the (110) QW's are given as lines.

This mixing has its fingerprint on the optical anisotropy, displayed in Fig. 4(b). The experimental anisotropy is smaller than the calculated anisotropy for the TW transition. This shows that, in the TW exciton, the valence-band state has a stronger admixture of the (001) QW states than the TW valence-band state. The reason for this finding is the shape of the conduction-band TW state, which is extended more into the (001) QW than the valence-band TW state [Fig. 4(c)].

While increasing the (110) QW width, the TW states shift from the (001) QW into the (110) QW due to the change of the balance between the (001) and (110) transition energies. The lower of the QW states contributes more to the TW state compared to the upper one. As a consequence, the optical anisotropy of the TW state increases with increasing (110) QW width, but stays between the (001) QW anisotropy of zero and the (110) QW anisotropy of about 0.74 .

\section{Asymmetric structures with $\mathrm{Al}_{x} \mathrm{Ga}_{1-x} \mathrm{As}$ in the (001) wells and optimization of the wire confinement}

To further confine the electronic states, a stronger asymmetric QW structure with $\mathrm{Al}$ in the (001) QW has to be implemented, as discussed already in the presented analytical model (Sec. II A). Assuming a given width of the overgrown (110) QW, we optimize the WCE by a variation of the width and the aluminum content of the (001) QW's. The (001) barrier width is chosen to be large enough to avoid superlattice effects for the TW states. The barrier composition was fixed at $\mathrm{Al}_{0.3} \mathrm{Ga}_{0.7} \mathrm{As}$. For a small $\mathrm{QW}$ width, a higher aluminum content increases the WCE. ${ }^{13,28}$ However, care has to be taken to avoid a type-II structure induced by the $\Gamma$-X crossing at $42 \%$ aluminum content. The largest energy separation between the TW-transition and the transitions in the (001) and (110) QW's is obtained, when the transition energies in both QW's are equal. This condition determines the optimum aluminum content to be used in the (001) QW's of a given width.

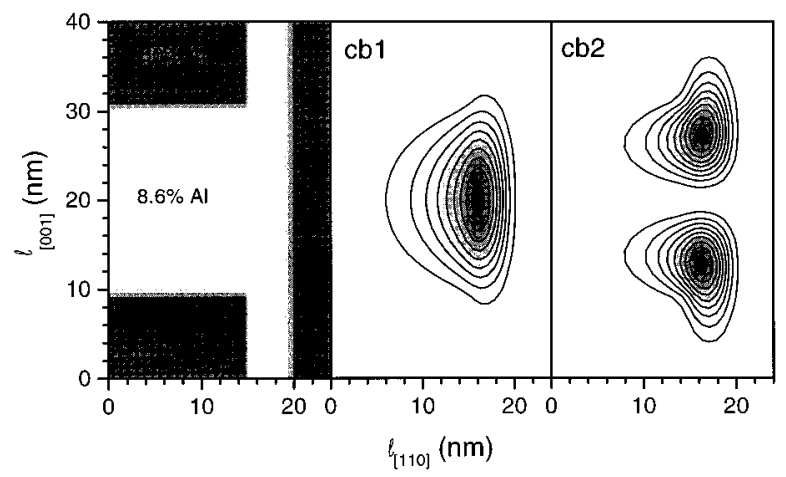

FIG. 6. TW states in a T-shaped structure consisting of a 20-nm $\mathrm{Al}_{0.086} \mathrm{Ga}_{0.914} \mathrm{As} / 20$-nm $\mathrm{Al}_{0.3} \mathrm{Ga}_{0.7} \mathrm{As}$ (001) MQW overgrown by a 4-nm (110) GaAs QW. The shape of the structure is depicted in the left upper picture. The contours of constant probability are plotted for the two conduction-band TW states (cb1) and (cb2). 
TABLE II. Calculated structural parameters, transition energies, and electron and hole confinement energies of the optimized T-shaped wire structures.

\begin{tabular}{lccccc}
\hline \hline $\begin{array}{l}(110) \text { QW } \\
\text { width }(\mathrm{nm})\end{array}$ & $\begin{array}{c}(001) \text { QW } \\
\text { width }(\mathrm{nm})\end{array}$ & $\begin{array}{c}\text { Al } \\
(\%)\end{array}$ & $\begin{array}{c}\text { Transition } \\
\text { energy }(\mathrm{eV})\end{array}$ & $\begin{array}{c}\text { Electron confinement } \\
(\mathrm{meV})\end{array}$ & $\begin{array}{c}\text { Hole confinement } \\
(\mathrm{meV})\end{array}$ \\
\hline 3 & 16 & 12.4 & 1.643 & 29 & 4.8 \\
4 & 18 & 9.25 & 1.609 & 26 & 3.2 \\
5 & 20 & 7.1 & 1.587 & 23 & 2.7 \\
7 & 24 & 4.5 & 1.561 & 17 & 1.4 \\
10 & 30 & 2.65 & 1.544 & 11 & 0.6 \\
\hline \hline
\end{tabular}

We now consider structures with different (001) QW widths and the optimum aluminum content. The effect of an increasing well width can be regarded as an increase of the quantization length along the [001] direction for the TW state, which is reducing the TW quantization energy along the [001] direction. At even larger (001) QW widths, a second TW state can be bound to the intersection. This reduces the effective WCE of the first TW state, which then has to be taken as its energy separation to the second TW state. The optimum WCE is thus established just at the (001) QW width at which the second TW state becomes confined. This behavior resembles the numerical results, which are given in Fig. 5 for overgrown (110) GaAs QW widths of 3, 4, 5, 7, and $10 \mathrm{~nm}$. The energies of the TW states (squares) in the conduction and valence bands and the TW transition energy are displayed as a function of the (001) QW width, using the optimum aluminum content for each width. The energies in the corresponding (110) QW's are given as lines, indicating the level of the TW barriers. The general trend is an increase of the WCE with increasing (001) QW width.

At a certain width, a second TW state (triangles) appears in the conduction band below the (110) QW energy. The TW states in a T-shaped structure with two TW states in the conduction-band are shown in Fig. 6. The first conductionband TW state is well confined at the intersection and exhibits no nodes, whereas the second conduction-band TW state shows a node along the [001] direction. It is thus a second state along the [001] quantization, in agreement with the pre-

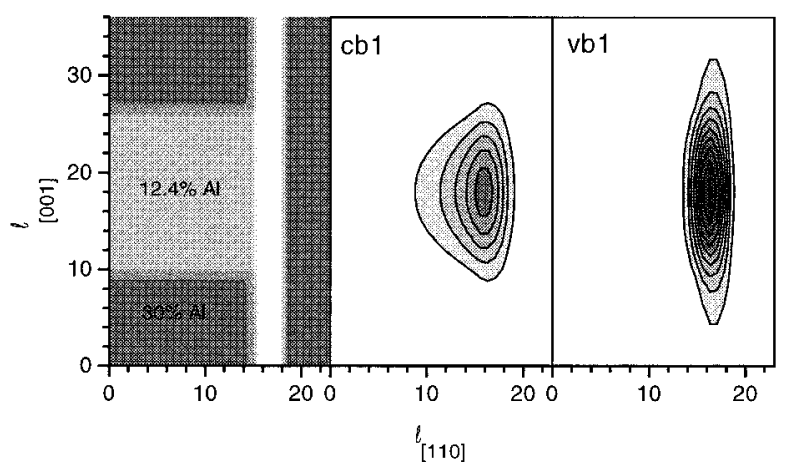

FIG. 7. TW states in the optimized T-shaped structure consisting of a 16-nm $\mathrm{Al}_{0.124} \mathrm{Ga}_{0.876} \mathrm{As} / 20-\mathrm{nm} \mathrm{Al}_{0.3} \mathrm{Ga}_{0.7} \mathrm{As}$ (001) MQW overgrown by a 3-nm (110) GaAs QW and an $\mathrm{Al}_{0.3} \mathrm{Ga}_{0.7} \mathrm{As}$ barrier. The shape of the structure is shown at left, and the contours of the constant probability are plotted in the middle and right graphs for the conduction- and valence-band TW states, respectively. vious discussion. The onset of confinement of the second TW state in the conduction band marks the optimal (001) QW width for a given (110) QW width. The resulting parameters of the T-shaped structures with the optimal design are listed in Table II. The WCE in these optimized structures is about twice the WCE of a symmetric structure of equal (110) QW width [compare with Fig. 2(b)].

For the optimized 3-nm T-shaped structure, the geometrical shape and the TW states are given in Fig. 7. The states
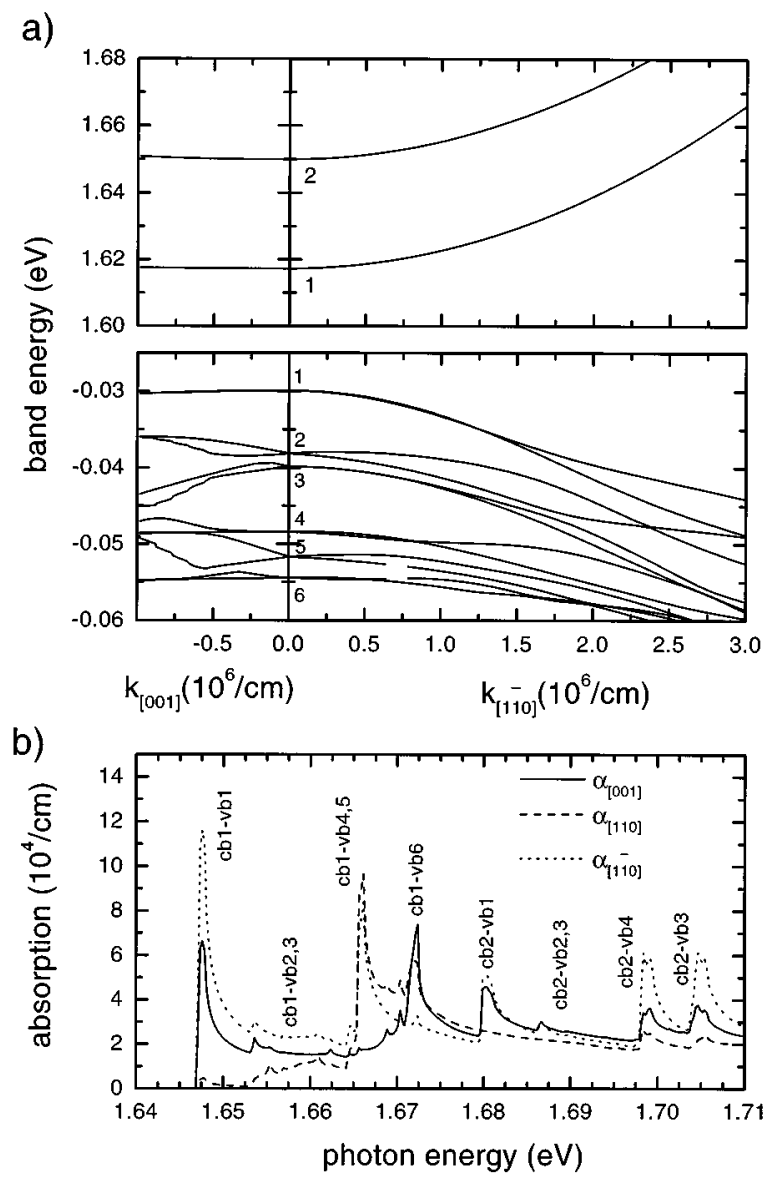

FIG. 8. Calculated subband dispersions and interband absorption in the optimized 3-nm structure. (a) Subband dispersions of the lowest two conduction subbands and the highest six valence subbands from the zone center in the direction [1피 along the wire and in the direction [001] along the wire array. (b) Absorption $\alpha_{[h k l]}$ for the three principal light polarizations, as indicated. The transitions are labeled according to the involved subband states. 
are stronger confined at the intersection compared to the TW states in the corresponding symmetric structure (Fig. 3). Especially for the valence-band TW state, the confinement is improved.

The calculated subband dispersions and interband absorption of this structure are shown in Fig. 8. As these results also are calculated using an array of $\mathrm{T}$ intersections in the [110] direction by cutting the (001) MQW in a depth of 15 $\mathrm{nm}$, the unbound states are not the same as in the real structure.

The subband dispersion [Fig. 8(a)] along the wire direction $\left(k_{[1 \bar{\Gamma}]]}\right)$ shows a nearly parabolic shape for the conduction-band states. In contrast, the valence-band states show a strong nonparabolicity of the dispersion along the wire due to the mixing of the heavy- and light-hole valence bands. The broken inversion symmetry of the $\mathrm{T}$ structure leads to a lifting of Kramer's degeneracy outside the zone center. The decoupling of TW states confined at adjacent QW's of the MQW structure results in flat dispersions of the lowest conduction subband and the highest valence subband along the MQW direction $\left(k_{[001]}\right)$. The higher valence-band states show a strong dispersion along this direction, due to their extended character.

The calculated interband absorption [Fig. 8(b)] for the three principal light polarizations along [001], [110], and [110] shows the typical peaks of the joint density of states at a quantum-wire subband edge of the energy $E_{n}$ proportional to $\left(\hbar \omega-E_{n}\right)^{-1 / 2}$. Above the fundamental transition between the confined TW states (cb1-vb1), several transitions to higher valence subbands appear, that have a different polarization as the fundamental transition. This reflects the different mixtures of heavy and light holes in the higher valence subbands. In a realistic structure, which is extended along the [110] MQW direction, this is expected to show up as a continuum instead of separated transitions, since the higher valence subbands are not confined at the intersection. Consequently, in the absorption of the structure, only the low confinement of the valence-band TW state will determine the spacing to the higher subband transitions. The Coulomb interaction is expected to influence these results by the mixing of the valence bands.

\section{EXPERIMENTAL REALIZATION OF THE OPTIMIZED STRUCTURES}

After the optimal design of the T-shaped structures of a given (110) GaAs QW width has been determined, as described in Sec. III, we grew two of these structures by the cleaved edge overgrowth technique ${ }^{4,7}$ using MBE. We selected structures with 3- and 5-nm overgrown well thicknesses. The (001) multiple-quantum-well structure for the 3 -nm overgrowth nominally consists of a $500-\mathrm{nm}$ GaAs buffer layer, 20 periods of $\left(12-\mathrm{nm} \mathrm{Al}_{0.116} \mathrm{Ga}_{0.895} \mathrm{As} / 20-\mathrm{nm}\right.$ $\mathrm{Al}_{0.3} \mathrm{Ga}_{0.7} \mathrm{As}$ ), and a 5-nm GaAs cap layer. The (001) multiple-quantum-well structure for the 5-nm overgrowth nominally consists of a $500-\mathrm{nm}$ GaAs buffer layer, 50 periods of (18-nm $\mathrm{Al}_{0.069} \mathrm{Ga}_{0.937} \mathrm{As} / 32-\mathrm{nm} \mathrm{Al}_{0.3} \mathrm{Ga}_{0.7} \mathrm{As}$ ), and a 10-nm GaAs cap layer. The barrier width was chosen to be large enough to avoid coupling between the TW states. The relative $\mathrm{Ga}$ and $\mathrm{Al}$ flux was adjusted to give a barrier composition of $\mathrm{Al}_{0.3} \mathrm{Ga}_{0.7} \mathrm{As}$. The III/V flux ratio was 15 , and the

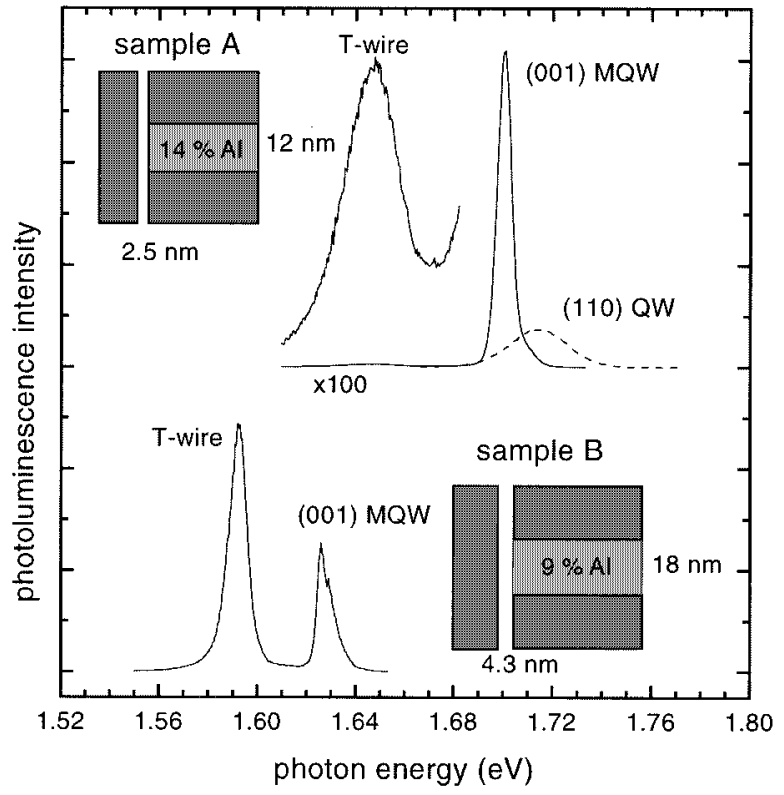

FIG. 9. PL of the T-wire samples $A$ and $B$ at 40-K lattice temperature, under edge excitation with $0.1 \mathrm{~W} / \mathrm{cm}^{2}$ at $632 \mathrm{~nm}$ (sample $A$ ) and $488 \mathrm{~nm}$ (sample $B$ ). The shape of the structures is schematically depicted in the inserts. The PL of the reference (110) QW for sample $A$ is also displayed (dashed line).

growth temperature was $700{ }^{\circ} \mathrm{C}$.

To grow a QW with low aluminum content, we used the digital alloy (DA) technique. During the growth of each well, the aluminum shutter was opened and closed ten (20) times for sample $A(B)$, which corresponds to a DA period of 0.12 $(0.09) \mathrm{nm}$. Since this is less than the interdiffusion length of $\mathrm{Ga}$ and $\mathrm{Al}$ during the growth, this procedure results in a homogeneous alloy. As the opening time for the aluminum shutter is comparable to its switching speed, the absolute aluminum content of the layer cannot be directly determined from the nominal shutter times of the MBE system. To compensate for the possible deviation, we measured the transition energy in the DA MQW's by low-temperature photoluminescence (PL) before the overgrowth. The width of the overgrown (110) GaAs QW was then adjusted to match its transition energy with the DA MQW transition energy. As we discussed in Sec. IIIC, this is important for a high WCE, whereas the width of the (001) QW is not critical (compare Fig. 5). To perform this matching, we have previously investigated the dependence of the transition energy in (110) QW's on the well width. ${ }^{26}$

For the overgrowth, the substrates of DA MQW's were lapped down to $90 \mu \mathrm{m}$ and cut into $5 \times 6 \mathrm{~mm}^{2}$ pieces along the (110) direction. They were remounted vertically on a special holder, cleaved inside the MBE chamber, and immediately overgrown on the cleaved edge along the (110) direction by a 2.5- (4.3-) nm GaAs QW for sample $A(B)$, a 20(32-) $\mathrm{nm} \mathrm{Al}_{0.3} \mathrm{Ga}_{0.7} \mathrm{As}$ barrier, and a $10-(1500) \mathrm{nm} \mathrm{GaAs}$ cap. The growth temperature was $460(500){ }^{\circ} \mathrm{C}$ and the III/V flux ratio was 30 for sample $A(B)$.

In Fig. 9, we show the PL of samples $A$ and $B$ at a $40-\mathrm{K}$ lattice temperature, excited on the overgrown edge with about $0.1 \mathrm{~W} / \mathrm{cm}^{2}$ at 633 and $488 \mathrm{~nm}$, respectively. The PL of the (001) MQW transition and the TW transition are sepa- 
rated by $54 \mathrm{meV}$ for sample $A$, and $37 \mathrm{meV}$ for sample $B$. The difference in the PL efficiency of the TW transition in the two samples is due to the different overgrowth parameters mentioned above. Especially the thicker GaAs cap layers on sample $B$ is leading to a higher PL yield.

A reference (110) QW of the same nominal width and barrier composition as in sample $A$ was grown on a planar (110) substrate after the cleaved edge overgrowth of sample A. Since we could use the same growth calibration, the reference QW is expected to be very similar to the overgrown QW. The PL of this reference QW is shown as a dotted line in Fig. 9. Its transition energy coincides approximately with the transition energy of the (001) MQW, which confirms the accuracy of the design and growth of the (110) QW after the characterization of the (001) MQW transition energy. The larger inhomogeneous width of the (110) QW transition (24 $\mathrm{meV}$ ) compared to the (001) MQW transition (5 meV) is due to the narrow well width of the (110) QW compared to the (001) MQW combined with a higher interface roughness during growth on the (110) surface compared to the (001) surface. ${ }^{26}$ The inhomogeneous width of the TW transition is $20 \mathrm{meV}$. A calculation of the dependence of the TW and (110) QW transition energies on the (110) QW width gives about $40(60) \mathrm{meV} / \mathrm{nm}$, respectively. The effective interface roughness of the (110) QW can thus be estimated to $0.6 \mathrm{~nm}$. The ratio between the two calculated dependencies corresponds to the ratio of the inhomogeneous widths of TW and (110) QW PL, if we neglect the small width of the (001) QW PL. The experimental ratio of $20 / 25$ is in reasonable agreement with the calculated ratio 40/60.

To compare the experimental WCE with theory, we have calculated the WCE for the two structures, giving 39 (28) meV for sample $A(B)$. The experimental WCE thus supersedes the calculated ones by about $25 \%$. Such an increased WCE was attributed previously ${ }^{18}$ to an enhanced exciton binding energy of the TW states compared to the QW states. The exciton binding energies calculated by Chang, Chang, and $\mathrm{Esaki}^{2}$ for sample $A(B)$ amount to about $20(16) \mathrm{meV}$, which is 10 (7) $\mathrm{meV}$ more than the exciton binding energy in the corresponding (001) QW's. This is less than the observed difference between the calculated and experimentally determined WCE's. However, other mechanisms such as strain shifts or unequal overgrowth on well and barrier regions of the (001) MQW could also account for the discrepancy. Additionally, the $\mathbf{k} \cdot \mathbf{p}$ model used in the calculation could not be precise enough to determine the WCE to the accuracy of some meV, especially for thin overgrown QW's. The observed WCE's are the highest reported up to now, which confirms the successful optimization of the T-shaped structures for maximum WCE.

\section{CONCLUSION}

In conclusion, we calculated and optimized the confinement energies for electrons and holes at the $\mathrm{T}$ intersection of two QW's. We have shown that, for an optimized design, $e-h$ pair WCE's of $40 \mathrm{meV}$ are achievable. The valence-band WCE is found to be between 2 and $5 \mathrm{meV}$ in the considered well-width range. The Coulomb interaction, which was neglected in the calculations, results in an enhanced confinement of the holes at the intersection. This is because the exciton binding energy is larger than the hole subband separation, and thus mixes different hole subbands. The successful optimization of the T-shaped structures is confirmed by the growth of an optimized structure, performing a confinement energy of $54 \mathrm{meV}$, which is significantly higher than previously reported.

\section{ACKNOWLEDGMENTS}

The authors want to thank C.B. Sørensen and D. Birkedal for helpful discussions. This work was supported by the Danish Ministries of Research and Industry in the framework of CNAST (Center for Nanostructures).
${ }^{1}$ E. Kapon, D. M. Wang, and R. Bhat, Phys. Rev. Lett. 63, 430 (1989).

${ }^{2}$ Y-C. Chang, L.L. Chang, and L. Esaki, Appl. Phys. Lett. 47, 1324 (1985).

${ }^{3}$ L. Pfeiffer et al., Appl. Phys. Lett. 56, 1697 (1990).

${ }^{4}$ D. Gershoni et al., Phys. Rev. Lett. 65, 1631 (1990).

${ }^{5}$ H. L. Störmer et al., Appl. Phys. Lett. 58, 726 (1991).

${ }^{6}$ A. R. Goñi et al., Appl. Phys. Lett. 61, 1956 (1992).

${ }^{7}$ W. Wegscheider et al., J. Cryst. Growth 150, 285 (1995).

${ }^{8}$ R. D. Grober et al., Appl. Phys. Lett. 64, 1421 (1994).

${ }^{9}$ T. D. Harris et al., Appl. Phys. Lett. 68, 988 (1996).

${ }^{10}$ W. Wegscheider et al., Phys. Rev. Lett. 71, 4071 (1993).

${ }^{11}$ W. Wegscheider et al., Semicond. Sci. Technol. 9, 1933 (1994).

${ }^{12}$ W. Wegscheider, L.N. Pfeiffer, K.W. West, and R.E. Leibenguth, Appl. Phys. Lett. 65, 2510 (1994).

${ }^{13}$ T. Someya, H. Akiyama, and H. Sakaki, Appl. Phys. Lett. 66, 3672 (1995).

${ }^{14}$ E. C. Valadares, Phys. Rev. B 46, 3935 (1992).

${ }^{15}$ R. K. Hayden et al., Semicond. Sci. Technol. 9, 298 (1994).
${ }^{16}$ D. S. Citrin and Y.-C. Chang, Phys. Rev. B 40, 5507 (1989).

${ }^{17}$ T. Someya, H. Akiyama, and H. Sakaki, Phys. Rev. Lett. 74, 3664 (1995).

${ }^{18}$ T. Someya, H. Akiyama, and H. Sakaki, Phys. Rev. Lett. 76, 2965 (1996).

${ }^{19}$ M. H. Degani and O. Hipólito, Phys. Rev. B 35, 9345 (1987).

${ }^{20}$ E. Rinaldi et al., Phys. Rev. Lett. 73, 2899 (1994).

${ }^{21}$ J. M. Luttinger, Phys. Rev. 102, 1030 (1956).

${ }^{22}$ G. Edwards, E. Valdares, and F. W. Sheard, Phys. Rev. B 50, 8493 (1994).

${ }^{23}$ S. Nojima, Phys. Rev. B 47, 13535 (1993).

${ }^{24}$ P. Lawaetz, Phys. Rev. B 4, 3460 (1971).

${ }^{25}$ J.-B. Xia, Phys. Rev. B 43, 9856 (1991).

${ }^{26}$ H. Gislason, C. Sørensen, and J. M. Hvam, Appl. Phys. Lett. 69, 800 (1996).

${ }^{27}$ H. Akiyama, T. Someya, and H. Sakaki, Phys. Rev. B 53, R10 520 (1996).

${ }^{28}$ W. Langbein (unpublished). 